\title{
Dor no Paciente com Síndrome Pós-COVID-19
}

\author{
Pain in the Patient with Post-COVID-19 Syndrome
}

Correspondence addresses:

Dr. Anita Perpetua Carvalho Rocha de Castro

anitaperpetuacre@yahoo.com.br

Received: April 27, 2021

Revised: May 15, 2021

Accepted: June 1st, 2021

Published: June 30, 2021

Data Availability Statement: All relevant data are within the paper and its Supporting Information files.

Funding: This work was the result of authors' initiative. There was no support of research or publication funds.

Competing interests: The authors have declared that no competing interests exist.

Copyright

(C) 2021 by Santa Casa

de Misericórdia da Bahia.

All rights reserved.

ISSN: 2526-5563

DOI: 10.35753

\author{
Anita Perpetua Carvalho Rocha de Castro ${ }^{1 *}$, Jedson dos Santos Nascimento ${ }^{1}$, \\ Mariana Camargo Palladini ${ }^{1}$, Lia Rachel Chaves do amaral Pelloso ${ }^{1}$, Marcio \\ Henrique Lopes Barbosa ${ }^{1}$ \\ ${ }^{1}$ Anesthesiology Service of Santa Izabel Hospial; Salvador, Bahia, Brazil
}

A COVID-19 é uma doença infecciosa que afeta as pessoas de diferentes maneiras. A maioria dos infectados apresenta sintomas leves a moderados, de curta duração e não precisa ser hospitalizada. Entretanto, alguns indivíduos cursam com sintomas mais prolongados e desenvolvem a síndrome pós-COVID-19. Esta síndrome é representada por uma ampla gama de novas alterações de saúde, as quais são recorrentes ou contínuas em pessoas que foram infectadas com o vírus SARS-Cov-2. Há relatos de pessoas experimentando cansaço ou fadiga, desnutrição, dificuldade de concentração, anosmia ou ageusia, tontura, taquicardia, palpitação, dispneia, tosse, transtornos do humor, fibrose pulmonar, insuficiência renal crônica e dor. $O$ objetivo deste artigo é fazer uma revisão sobre a dor no paciente portador de síndrome pós-COVID-19, discutindo acerca dos mecanismos envolvidos e do seu impacto na qualidade de vida do indivíduo. Pacientes com síndrome pós-COVID-19 podem apresentar dor, que parece ser mais prevalente naqueles indivíduos que estiveram internados em unidade de terapia intensiva (UTI). Os pacientes neste contexto são submetidos a inúmeros fatores de risco, como: dor aguda mal tratada, insultos neurológicos, múltiplas comorbidades e alterações relacionadas à necessidade de ventilação prolongada, imobilização, bloqueio neuromuscular, posição pronada e sepse. Isolamento, internamento em UTI e o medo de morrer têm sido uma realidade para muitos pacientes, os quais apresentam sequelas psicológicas graves e grande morbidade. A condução do paciente e da dor na síndrome pósCOVID-19 deve ser multiprofissional. Só através da atuação de uma equipe formada por médicos de diferentes especialidades, fisioterapeutas, nutricionistas, psicólogos e fonoaudiólogos é possível atender à necessidade desses pacientes. O acompanhamento dos indivíduos portadores de síndrome pós-COVID-19 é essencial, não apenas para monitorar e tratar esses pacientes, mas também para compreender totalmente o impacto a longo prazo da infeç̧ão por SARS-Cov-2. Palavras-chave: Síndrome Pós-COVID-19; Dor; Sintomas.

COVID-19 is an infectious disease that affects people in different ways. Most infected patients have mild to moderate symptoms that are short-lived and do not need hospitalization. However, some individuals have more prolonged symptoms and develop the post-COVID-19 syndrome. This syndrome presents a wide range of new health changes, which are recurrent or ongoing in people who had the disease. There are reports of people experiencing tiredness or fatigue, malnutrition, difficulty concentrating, anosmia or ageusia, dizziness, tachycardia, palpitation, dyspnea, cough, mood disorders, pulmonary fibrosis, chronic renal failure, and pain. This article aims to review pain in patients with post-COVID-19 syndrome, discussing the mechanisms involved and their impact on the quality of the person's life. Patients 
with the post-COVID-19 syndrome may experience pain, which seems to be more prevalent in those admitted to the ICU. In this context, patients are subjected to numerous risk factors, such as poorly treated acute pain, neurological insults, multiple comorbidities, and changes related to the need for prolonged ventilation, immobilization, neuromuscular block, prone position, and sepsis. Isolation, admission to ICU, and the fear of death are the reality for many patients who had severe psychological sequelae and morbidity. The management of the patient and pain in post-COVID-19 syndrome must be multi-professional. Only through the performance of a team formed by doctors from different specialties, physiotherapists, nutritionists, psychologists, and speech therapists is it possible to meet the needs of these patients. The follow-up of individuals with post-COVID-19 syndrome is essential to understand the long-term impact of SARS-Cov-2's infection and to monitor and treat these patients.

Keywords: Post-COVID-19 Syndrome; Pain; Symptoms.

\section{Introdução}

A COVID-19 é uma doença infecciosa que afeta as pessoas de diferentes maneiras. A maioria dos pacientes infectados apresentam sintomas leves a moderados e não precisam ser hospitalizados. Dentre os sintomas mais comuns vivenciados na fase aguda encontramse febre, tosse seca e cansaço, entretanto, alguns pacientes desenvolvem dor na garganta, diarreia, conjuntivite, dor de cabeça, perda de paladar ou olfato. Sintomas mais graves incluem dificuldade de respirar, compressão torácica e perda da fala ou do movimento. O SARS-Cov-2 é um vírus que tem como alvo principal o sistema respiratório humano e que os sintomas da infecção por este vírus geralmente aparecem após um período de incubação. Embora os principais sintomas durante a doença COVID-19 sejam agudos e a maioria dos pacientes se recupere totalmente, uma parcela destes experimentam danos mais persistentes, ${ }^{1}$ o que a literatura tem denominado de síndrome pós-COVID-19. Esta é uma complicação decorrente da infecção pelo SARS-Cov-2, considerada como uma condição inflamatória difusa e multissistêmica.

A síndrome pós-COVID-19 é representada por uma ampla gama de novas alterações de saúde, as quais são recorrentes ou contínuas em pessoas que foram infectadas com o vírus SARS-Cov-2. Geralmente este quadro persiste por mais de quatro semanas após o evento inicial, mas podem estar presentes mesmo em pessoas que não apresentaram sintomas quando foram infectadas. A síndrome pós-COVID-19 pode ter diferentes tipos de combinações de problemas e se prolongar por longos períodos de tempo. Há relatos de pessoas experimentando cansaço ou fadiga, desnutrição, dificuldade de concentração ("névoa do cérebro"), anosmia ou ageusia, tontura, taquicardia, palpitação, dispneia, tosse, transtornos do humor como depressão e/ou ansiedade, fibrose pulmonar, insuficiência renal crônica e dor. Acreditase que essa síndrome seja semelhante à encefalomielite miálgica (EM) e à síndrome da fadiga crônica (SFC), síndromes que ocorrem devido a desregulação do sistema imunológico e do sistema nervoso autônomo, caracterizadas por recaída significativa após esforço físico, cognitivo ou emocional de qualquer tipo, resultando em comprometimento funcional significativo acompanhado por um nível patológico de fadiga. As alterações metabólicas observadas incluem redução no suprimento de oxigênio muscular induzida por exercício físico, acidose no músculo esquelético ou desregulação de prótons, captação prejudicada de glicose e níveis reduzidos de trifosfato de adenosina nas células do músculo esquelético durante ou após o exercício. Outro elemento importante neste processo é a presença de autoanticorpos do receptor Beta-2-adrenérgico e do receptor da acetilcolina M3, uma vez que a remoção desses autoanticorpos por aférese de IgG leva a melhora da fadiga descrita. A fadiga mental e a dificuldade de concentração podem ser decorrentes da redução do fluxo sanguíneo cerebral secundária à vasoconstricção simpática excessiva na presença de receptores beta-2 disfuncionais. ${ }^{2}$ 
Pacientes com síndrome pós-COVID-19 referem dor articular, dor abdominal, dor no peito, dor de cabeça, dor muscular e dor de origem neuropática, sintomas que também pioram após atividades físicas ou mentais. Estes sintomas álgicos são mais comuns em pacientes que necessitaram de internamento em unidade de terapia intensiva (UTI). Dados recentes do Reino Unido, colhidos entre fevereiro e abril de 2020 , sugerem que $17 \%$ dos internados em unidades hospitalares requerem suporte avançado ou cuidados em UTI. Destes, mais de $50 \%$ necessitam de ventilação mecânica. ${ }^{3}$ Não se tem dados específicos relacionados aos pacientes portadores de COVID-19 quanto à prevalência de dor crônica decorrente desta patologia, entretanto, acredita-se que os dados não sejam diferentes daqueles observados em pacientes com síndrome pós-cuidados intensivos. As estimativas da prevalência de dor crônica após internamento na UTI variam de $14 \%$ a $77 \%$, dependendo da escala de tempo, método de medição e população. ${ }^{4}$ É provável que aqueles pacientes que apresentaram a forma grave da COVID-19 tenham uma maior chance de desenvolver dor crônica.

O objetivo deste artigo é fazer uma revisão sobre a dor no paciente portador de síndrome pós-COVID-19, discutindo acerca dos mecanismos envolvidos e do seu impacto na qualidade de vida do indivíduo.

\section{Por que a Dor faz Parte da Síndrome Pós- COVID-19?}

Pacientes com síndrome pós-COVID-19 podem apresentar dor, a qual acredita-se ser mais prevalente naqueles indivíduos que estiveram internados em UTI. Os pacientes neste contexto são submetidos a inúmeros fatores de risco, como: dor aguda mal tratada, insultos neurológicos, múltiplas comorbidades e alterações relacionadas à necessidade de ventilação prolongada, imobilização, bloqueio neuromuscular, posição pronada e sepse.
O tratamento da dor na UTI é um desafio. Trabalhos demonstram que apesar da existência de inúmeros protocolos ainda é negligenciada e subtratada. Pacientes criticamente enfermos sofrem procedimentos de aspiração, mobilização, drenagens, punções arteriais, procedimentos estes que são potencialmente dolorosos. ${ }^{5}$ No contexto da pandemia, observouse uma sobrecarga de trabalho da equipe e uma indisponibilidade de analgésicos em alguns serviços. É provável que isto tenha se traduzido em uma maior exposição à dor.

Um número grande de sobreviventes de COVID-19 internados em UTI foi submetido à sedação, imobilização e ventilação por um período prolongado. ${ }^{6}$ Além disto, fizeram uso de corticosteroides e de bloqueadores neuromusculares, elementos que representam fator de risco para o desenvolvimento de fraqueza muscular adquirida, miopatia e polineuropatia, quadros conhecidos como fraqueza adquirida após o internamento em UTI, miopatia do paciente crítico e polineuropatia do paciente crítico. Embora a dor não seja o sintoma principal destas condições clínicas, a fraqueza, a perda muscular e o comprometimento neurológico podem levar ao rápido descondicionamento físico, à dor articular, à síndrome dolorosa miofascial e à dor de origem neuropática.

Dor articular tem sido descrita em pacientes que apresentaram COVID-19. Sabe-se que a artrite reativa (AR) pode ocorrer após infecções virais e/ou bacterianas, sendo mais comum quatro semanas após o quadro inicial. $\mathrm{O}$ envolvimento articular pode ser mono ou poliarticular, mostrar-se com entesite e dactalite e acometer articulações localizadas nos membros superiores ou inferiores. É importante ressaltar que a AR tem inúmeros diagnóstico diferenciais como artrite gotosa, artrite séptica, artrite reumatoide e outras artropatias soronegativa, o que implica na necessidade de realização de testes específicos para melhor esclarecimento diagnóstico. Embora o número de casos descritos seja pequeno, é preciso 
estar atento a esta possibilidade, pois isto pode impactar na utilização de estratégias específicas e em uma evolução mais favorável do paciente. ${ }^{7}$

A dor neuropática após infecção por COVID-19 é uma realidade e tem origem após acometimento do sistema nervoso central ou periférico. Pacientes infectados por SARSCov-2 apresentam este sintoma por diferentes mecanismos: efeito imunológico desencadeado pelo SARS-Cov-2, ação direta do vírus ou como consequência do tratamento da própria doença infecciosa. Pacientes com COVID-19 tem apresentado encefalite, encefalomielite, acidente vascular cerebral isquêmico, hemorragia intracraniana e trombose venosa cerebral. Doenças do sistema nervoso periférico incluem síndrome de Guillain-Barre (SGB) e suas variantes. A SGB é uma polirradiculopatia aguda imunomediada com uma ampla gama de manifestações clínicas.

Insuficiência respiratória neuromuscular ocorre em até $30 \%$ dos pacientes, levando a ventilação mecânica e internamento em UTI, elementos que são considerados fatores de risco para o desenvolvimento de dor crônica na síndrome pós-COVID-19. Os mecanismos fisiopatológicos envolvidos ainda não estão bem estabelecidos, mas acredita-se que assim como em outros quadros de paralisia parainfecciosa, este processo seja imunomediado, embora a possibilidade de um dano viral direto não tenha sido afastada, uma vez que há evidências de que o SARS-Cov-2 tenha características neurotrópicas e neuroinvasivas. ${ }^{8}$

Outras formas de neuropatia periférica também têm sido relatadas. Bahouth e colaboradores $^{9}$ descreveram um caso clínico de um paciente que desenvolveu doença neuromuscular na ausência de outro fator causal, caracterizada pela presença de redução da força muscular, parestesia em região posterior da nádega e em membro inferior esquerdo, até o pé. Ao exame físico este apresentava ausência de dorsoflexão ativa, flexão plantar, eversão ou inversão do tornozelo esquerdo, com amplitude de movimento ativa normal no tornozelo direito. Mantinha capacidade normal de flexionar e estender ambos os joelhos. Esta descrição não é compatível com SGB clássica ou suas variantes, o que fortalece a possibilidade de outra etiologia para o quadro descrito.

Um dos pilares do suporte respiratório durante a pandemia de COVID-19 é a posição pronada. ${ }^{10}$ Este posicionamento pode implicar em comprometimento da função neural. Há descrições de lesões do plexo braquial, subluxações articulares e de síndrome dolorosa miofascial, condições estas que frequentemente estão associadas a presença de dor. Zhou e Siao, ${ }^{11}$ em uma publicação recente, descreveram dois casos clínicos de pacientes que desenvolveram meralgia parestésica após a posição pronada para suporte ventilatório em pacientes com COVID-19. A meralgia parestésica é uma neuropatia causada por compressão do nervo cutâneo femoral lateral ao nível da espinha ilíaca antero-superior ou ligamento inguinal. Esta é comumente associada a diabetes mellitus, obesidade e uso de roupas justas, mas também pode ser iatrogênica, causada pelo posicionamento durante cirurgias de coluna. Geralmente os pacientes apresentam como sintomas clássicos dor, parestesia ou queimação e diminuição da sensibilidade tátil e dolorosa na face antero-lateral da coxa.

\section{Impacto Psíquico Associado à Dor na Síndrome Pós-COVID-19}

O impacto psicológico da dor, seja ela aguda ou crônica tem sido alvo de interesse crescente na prática clínica. Na pandemia de COVID-19 este interesse tem sido crescente. Mesmo em indivíduos saudáveis há uma recomendação por parte das instituições de saúde e instituições governamentais de isolamento social, além de uma preocupação quanto ao contágio, ao desenvolvimento da forma grave do COVID-19 e à possibilidade de morrer. Esta preocupação por si só causa adoecimento, 
o qual é maior naqueles que perderam seus entes queridos ou que estiveram internados em UTI. Sequelas psicológicas graves têm sido relatadas em sobreviventes de UTI que desenvolveram síndrome do desconforto respiratório agudo. Destes, 30\% apresentaram transtorno de estresse pós-traumático. ${ }^{12}$ Nos sobreviventes da COVID-19, acredita-se que este impacto seja ainda maior, uma vez que os pacientes internados na UTI durante a pandemia foram expostos à proibição do contato com a família, ao isolamento proporcionado pelos equipamentos de proteção individual e ao distanciamento da própria equipe de saúde. $\mathrm{Li}_{1}{ }^{13}$ ao avaliar pacientes em reabilitação identificou, dentre outros sintomas, a presença de transtorno de ansiedade nesta população e ponderou a necessidade de um olhar mais cuidadoso para o adoecimento psíquico neste contexto. O que se observa é que a dor tem sido considerada como um elemento central na etiologia dos transtornos psíquicos relacionados ao COVID-19, sejam eles transtorno de estresse pós-traumático, transtorno de ansiedade ou depressão.

No momento, os recursos de saúde estão voltados para a vacinação contra o SARSCov-2 e para os atendimentos de linha de frente, entretanto, é importante lembrar que o controle adequado da dor e intervenções psíquicas precoces têm um impacto positivo na evolução dos pacientes. ${ }^{3}$

\section{A Condução do Paciente e da Dor na Síndrome Pós-COVID-19}

Pacientes com síndrome pós-COVID-19 devem ser abordados de forma multiprofissional. $\mathrm{O}$ primeiro passo é a realização de uma avaliação cuidadosa através de uma história clínica e exame físico detalhados, os quais são essenciais para a definição da melhor abordagem terapêutica a ser adotada. Comorbidades prévias como diabetes mellitus, hipertensão, doença renal ou doença cardíaca devem ser tratadas em conjunto com os sintomas de síndrome pós-COVID-19. Novos achados devem chamar a atenção para a possibilidade de desenvolvimento de novas patologias. A presença de sopros ou ritmo de galope ao exame físico pode sugerir envolvimento cardíaco, por exemplo. Testes neurológicos alterados podem indicar comprometimento cognitivo. ${ }^{2}$ Naqueles pacientes com dor, é fundamental a utilização de questionários que possam identificar a presença de componente neuropático. No Brasil os instrumentos mais frequentemente aplicados são a escala de dor de LANNS ("Leeds Assessment of Neuropathic Symptoms and Signs"), o questionário DN4 ("Douleur Neuropathique 4 Questions") e o "Neuropathic Pain Symptom Inventory", pois representam aqueles que primeiro foram traduzidos e validados para o português.

A fadiga e a falta de ar podem ser multifatoriais e ter em comum algumas características da síndrome da fadiga crônica descrita após outras infecções graves. Deve-se solicitar hemograma completo, exames radiológicos de tórax e/ou teste de função pulmonar para descartar anemia e doença pulmonar residual. Para indivíduos com dor torácica indica-se a medição da taxa de hemossedimentação, dosagem sérica de proteína $\mathrm{C}$ reativa e de troponina, juntamente com exames cardiológicos específicos, os quais irão afastar miopericardite ou síndrome coronariana aguda. Níveis elevados de ferritina podem indicar inflamação e um estado protrombótico continuado. Se o paciente foi diagnosticado com um evento trombótico, como uma embolia pulmonar, a anticoagulação deve ser continuada de acordo com as diretrizes atuais. ${ }^{2}$ Sintomas respiratórios, cardiológicos, psicológicos, neurológicos ou que envolvam o trato gastrointestinal persistentes devem implicar no encaminhamento ao especialista apropriado, seja ele pneumologista, cardiologista, psiquiatra, gastroenterogista, especialista em doenças infecciosas, neurologista ou nefrologista.

A dor crônica pós-COVID-19 pode afetar pacientes de qualquer idade, mas parece ser 
mais comum em pacientes mais velhos. ${ }^{3}$ Este dado é preocupante pois os sintomas álgicos aumentam o impacto psicossocial da síndrome pós-COVID-19. Neste contexto, deve-se buscar o tratamento adequado da dor, seja ela aguda ou crônica, através da utilização de medidas farmacológicas e não farmacológicas. Orienta-se seguir as recomendações da escada analgésica da Organização Mundial de Saúde, a qual diz que a dor leve deve ser tratada através da utilização de analgésico comum; a dor moderada através da aplicação de analgésico opioide fraco e a dor forte com o uso de analgésico opioide forte. Sempre que possível recomenda-se a associação de adjuvantes, os quais tem por objetivo otimizar a analgesia e/ou a prevenção e tratamento dos efeitos colaterais dos medicamentos principais. Pacientes portadores de dor neuropática necessitam de uma abordagem diferente. Neste caso, encontra-se indicado o uso de analgésicos antidepressivos, fármacos gabapentinoides e/ou anestésicos locais.

É importante observar que o processo de reabilitação deve envolver a participação de fisioterapeutas, nutricionistas, psicólogos e fonoaudiólogos, dentre outros profissionais que venham a compor a equipe multiprofissional. Pacientes com fadiga secundária à deficiência física podem se beneficiar com programas de exercícios de flexibilidade, exercícios aeróbicos e exercícios destinados a melhorar a marcha e o equilíbrio. ${ }^{14}$ Sintomas respiratórios significativos podem ser abordados através de programas de reabilitação pulmonar. Pacientes com sarcopenia e desnutrição beneficiam-se de uma avaliação e orientação nutricional. Dado o impacto psicológico do COVID-19 e o impacto socioeconômico de uma pandemia, é necessária uma avaliação psicológica completa. Os pacientes devem ser rastreados para complicações psiquiátricas agudas, como suicídio, bem como insônia, ansiedade, depressão e delírio.

\section{Conclusão}

Embora os efeitos agudos da COVID-19 tenham sido amplamente descritos, os efeitos em longo prazo são menos compreendidos. Pacientes infectados pelo SARS-CoV-2 enfrentam sintomas inesperados, os quais estão associados a grande impacto na sua qualidade de vida. A este fenômeno chama-se síndrome pósCOVID-19. O acompanhamento dos indivíduos portadores de síndrome dor pós-COVID-19 é essencial, não apenas para monitorar e tratar esses pacientes, mas também para compreender totalmente o impacto a longo prazo da infecção por SARS-Cov-2.

\section{Referências}

1. Max A, Schommers P, Stecher M, Dewald F, Gieselmann L, Gruell H, et al. Post-COVID síndrome in non-hospitalised patientes with COVID-19: a longitudinal prospective cohort study. The Lancet Regional Health 2021.

2. Scordo KA, Richmond MM, Munro N. PostCOVID-19 syndrome: theoretical basis, identification and management. AACN Adv Crit Care 2021;32(2):188-194.

3. Kemp HI, Corner E, Colvin LA. Chronic pain after COVID-19: implications for rehabilitation. Br J Anaesth 2020;125(4):436-440.

4. Kemp HI, Laycock H, Costello A, Brett SJ. Chronic pain in critical care survivors: a narrative review. Br J Anaesth. 2019;123:e372-e384.

5. Puntillo K.A., Max A., Timsit J.F. Determinants of procedural pain intensity in the intensive care unit: the Europain ${ }^{\circledR}$ study. Am J Respir Crit Care Med. 2014;189:39-47.

6. Yang $\mathrm{X}, \mathrm{Yu} \mathrm{Y}, \mathrm{Xu}$ J. Clinical course and outcomes of critically ill patients with SARSCoV-2 pneumonia in Wuhan, China: a singlecentered, retrospective, observational study. Lancet Respir Med. 2020;8:475-481.

7. J Ibtisam. Reactive arthritis after COVID-19. Cureus 2020;12(11):e11761.

8. Ottaviani D, Boso F, Tranquillini E, Gapeni I, Pedrotti G, Cozzio S, Guarrera GM, Giometto B. Early Guillain-Barré syndrome in coronavirus disease 2019 (COVID-19): a case report from an Italian COVID-hospital. Neurol Sci 2020;41(6):1351-1354. 
9. Bahouth S, Chuang K, Olson L, Rosenthal D. COVID-19 related muscle denervation atrophy. Sleletal Radiol 2021;50(8):1717-1721.

10. Phua J, Weng L, Ling L. Intensive care management of coronavirus disease 2019 (COVID-19): challenges and recommendations. Lancet Respir Med 2020;8:506-517.

11. Zhou Lan, Siao Peter. Lateral femoral cutaneous neuropathy caused by prone positioning to treat COVID-19-associated acute respiratory distress syndrome. Muscle \& Nerv 2021;1-3.
12. Nikayin S, Rabiee A, Hashem M. Anxiety symptoms in survivors of critical illness: a systematic review and meta-analysis. Gen Hosp Psychiatry. 2016;43:23-29.

13. Li J. Rehabilitation management of patients with COVID-19. Lessons learned from the first experiences in China. Eur J Phys Rehabil Med 2020;56(3):335-338.

14. Candan SA, Elibol N, Abdullahi A. Consideration of prevention and management of long-term consequences of post-acute respiratory distress syndrome in patients with COVID-19. Physiother Theory Pract 2020;36(6):663-668. 